\title{
MicroRNA-216a inhibits pancreatic cancer by directly targeting Janus kinase 2
}

\author{
SILIANG WANG, XIAODONG CHEN and MEIYUE TANG \\ Department of Medical Oncology, Shengjing Hospital of China Medical University, \\ Heping, Shenyang, Liaoning 110022, P.R. China
}

Received June 12, 2014; Accepted August 20, 2014

DOI: $10.3892 /$ or.2014.3478

\begin{abstract}
MicroRNA (miR)-216a expression is significantly downregulated in human pancreatic cancer, however, the underlying mechanism remains unknown. In the present study, we aimed to identify and characterize the direct target gene and potential function of miR-216a in pancreatic cancer cells. Bioinformatics analysis and dual-luciferase reporter gene assay showed that Janus kinase 2 (JAK2) was a direct target gene of miR-216a. Quantitative polymerase chain reaction and western blot analysis demonstrated that miR-216a decreased the mRNA and protein levels of JAK2 in pancreatic cancer cells. Phosphorylation of the signal transducer and activator of transcription 3 (STAT3) was also downregulated by miR-216a, whereas the anti-miR-216a treatment had an opposite effect. Treatment of pancreatic cancer cells with miR-216a significantly inhibited cell growth and promoted cell apoptosis. In addition, the downstream genes of JAK2/ STAT3, survivin and X-linked inhibitor of apoptosis protein, which are anti-apoptotic genes, were also decreased by miR-216a. Moreover, miR-216a overexpression markedly inhibited the JAK2/STAT3 signaling pathway and xenograft tumor growth in vivo. Compared with miR-216a treatment, anti-miR-216a treatment exhibited opposite effects throughout the entire experiment, confirming the inhibitory effect of miR-216a on pancreatic cancer by regulating the JAK2/STAT3 signaling pathway. The results provided evidence that miR-216a targeting JAK2 negatively regulated the development of pancreatic cancer cells and may be used to develop a miRNA-based therapeutic strategy against pancreatic cancer.
\end{abstract}

Correspondence to: Dr Siliang Wang, Department of Medical Oncology, Shengjing Hospital of China Medical University, No. 36 Sanhao Street, Heping, Shenyang, Liaoning 110022, P.R. China E-mail: shiliangwang_slw@163.com

Abbreviations: miR-216a, microRNA-216a; JAK2, Janus kinase 2; STAT3, signal transducer and activator of transcription 3; XIAP, $\mathrm{X}$-linked inhibitor of apoptosis protein; UTR, untranslated region

Key words: pancreatic cancer, microRNA, JAK2, STAT3

\section{Introduction}

Pancreatic cancer is the fourth leading cause of cancerassociated mortalities worldwide and is characterized by poor diagnosis and prognosis, and lack of efficient treatment (1). At the time of pancreatic cancer diagnosis, most of the patients have unresectable disease (2). Considerable efforts have been made to develop novel therapeutic treatments for this disease, but progress on this issue is limited. Application of gemcitabine alone or in combination with other chemotherapy drugs provides moderate benefit for survival, but is also accompanied by severe side-effects (3-6). Genetic screening of pancreatic cancer types determines various mutations in tumors, such as the most frequently mutated genes, including TP53, SMAD4 and Kras $(7,8)$. However, the therapeutic agents targeting these molecules have not achieved an optimum effect (9). Thus, identifying new molecular mechanisms involved in pancreatic tumorigenesis is necessary to provide novel insights for developing new and potent therapeutic options.

In recent years, microRNAs (miRNAs) have been shown to be involved in the regulation of a variety of human diseases (10). miRNAs are a subset of small, non-coding and endogenous RNA of 18-24 nucleotides in length that regulate gene expression by directly targeting the 3'-untranslated region (UTR) of mRNA leading to translational inhibition $(11,12)$. Recently, it was demonstrated that miRNAs are involved in a variety of cell processes, including apoptosis, proliferation and differentiation (10). Aberrant expression of miRNAs and their target genes have been identified in many cancer types, which are associated with oncogenesis and development (13). Aberrant expression of miRNAs is also found in pancreatic cancer (14). Findings of recent studies have shown that miR-216a, a member of the miR-216 family, is highly decreased in pancreatic cancer (15-17). However, the mechanism and function of miR-216a in pancreatic cancer remains to be determined.

Janus kinase 2 (JAK2)/signal transducer and activator of transcription 3 (STAT3) signaling pathway were shown to be involved in various types of cancer, including pancreatic cancer (18). STAT3 is a latent cytosolic transcription factor that is phosphorylated by JAK2, leading to STAT3 dimerization and nuclear localization to activate downstream genes $(19,20)$. The JAK2/STAT3 signaling pathway is involved in various cell processes, including proliferation and apoptosis, immune 
evasion and resistance, as well as angiogenesis in various types of cancer (21-23). Targeting JAK2/STAT3 may provide novel insight for pancreatic cancer therapy, such as lestaurtinib and INCB-18424, which are used in clinical trials to act on JAK2 (24).

Using bioinformatic algorithms, we identified JAK2 as a putative target gene of miR-216a. Considering the decreased expression level of miR-216a in pancreatic cancer, the interaction between JAK 2 and miR-216a may provide novel insights into the understanding of pancreatic cancer tumorigenesis as well as developing new therapeutic approaches. In the present study, the dual luciferase report assay was applied to verify whether JAK2 was a direct target of miR-216a. The effect of miR-216a overexpression or silencing on JAK2 and the downstream gene protein expression was determined in pancreatic cancer cells. The effect of miR-216a on cell growth and apoptosis in vitro and in vivo was also investigated. Our results showed that miR-216a targeting JAK2 negatively regulated pancreatic carcinogenesis, suggesting that miR-216a may be used as a potential target for the treatment of pancreatic tumorigenesis.

\section{Materials and methods}

Animals and cell lines. BALB/c nude mice weighing 25-30 g were purchased from the Medical Experimental Animal Center (Guangdong, China) and raised under standard conditions of room temperature and a dark-light cycle with free access to water. The animal experiments were approved and reviewed by the Institutional Animal Care and Use Committee of the China Medical University. The human Capan-2 and PANC-1 pancreatic cancer cell lines obtained from the Chinese Academy of Sciences (Shanghai, China) were cultured as per supplier's instructions. In brief, cells were grown in Dulbecco's modified Eagle's medium (DMEM; Invitrogen, Carlsbad, CA, USA) supplemented with $10 \%$ fetal bovine serum (Invitrogen) containing penicillin $(100 \mathrm{U} / \mathrm{ml})$ and streptomycin $(100 \mathrm{mg} / \mathrm{ml})$. Human embryonic kidney (HEK) 293 cells were cultured in DMEM supplemented with $10 \%$ fetal bovine serum and penicillin/streptomycin. The cells were cultured in an incubator at $37^{\circ} \mathrm{C}$ with $5 \% \mathrm{CO}_{2}$.

Luciferase reporter assay. The JAK2 3'-UTR was amplified using the primers: JAK2 3'-UTR forward, 5'-CCGCTCGAG AAGAAATGACCTTCATTCTGAGACCA-3' and reverse, 5'-AAGGAAAAAAGCGGCCGCTAAAGTAAGAAACTA TTTTCTTTTTAATCAA-3'. The mutated JAK2 3'-UTR was cloned using the primers: mut-JAK2 3'-UTR forward, 3'-GTA TCTATTTGTGGTGAATGATCGATCTAAATGGAACTA TCTCCAAA-5' and reverse, 5'-TTTGGAGATAGTTCC ATTTAGATCGATCATTCACCACAAATAGATAC-3' with a site-directed mutagenesis kit (Haoran Bio, Shanghai, China), in which the miR-216a binding site AGAGATT was mutated to CTCGCTC. The PCR products were then sub-cloned into a pGL3 Luciferase Reporter Vector (Promega, Madison, WI, USA) downstream of the luciferase gene. To examine whether JAK2 was a target of miR-216a, HEK293 cells were seeded into 96-well plates and co-transfected with $100 \mathrm{ng}$ pGL3 vector containing JAK2 3'-UTR or mutated forms with or without $20 \mathrm{nmol} / 1 \mathrm{miR}-216 \mathrm{a}$ mimic (GenePharma, Shanghai,

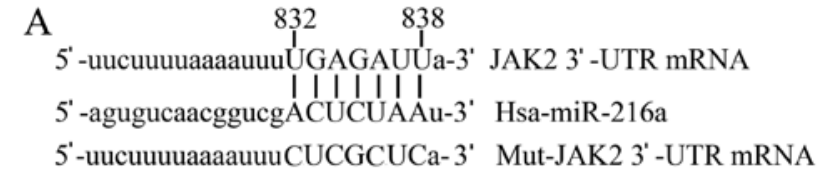

$\mathrm{B}$

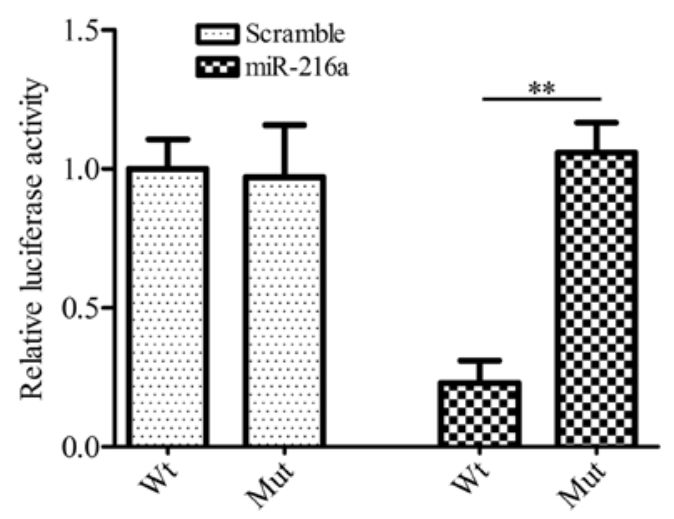

Figure 1. miR-216a directly targets 3'-UTR of JAK2. (A) Alignment of the miR-216a, wild-type and mutated 3'-UTR of JAK2. (B) The interaction of miR-216a with 3'-UTR of JAK2 was determined by luciferase reporter assays. Cells were co-transfected with a reporter plasmid containing the wild-type (WT) or mutant versions of 3'-UTR of JAK2 in the presence of $20 \mathrm{nmol} / 1 \mathrm{miR}-216 \mathrm{a}$ mimics or scramble miRNA, and incubated for $48 \mathrm{~h}$. ${ }^{* *} \mathrm{P}<0.01$. JAK2, Janus kinase 2 .

China) using Lipofectamine (Invitrogen). The transfected cells were cultured for $48 \mathrm{~h}$, and luciferase activity was measured using the Dual-Luciferase Reporter assay system (Promega).

Quantitative polymerase chain reaction ( $R T-q P C R)$. For mRNA analysis, total RNA from cells was extracted using TRIzol (Invitrogen) according to the manufacturer's instructions. Complementary DNA was synthesized by using M-MLV reverse transcriptase (Clontech, Palo Alto, CA, USA) according to the standard protocol. miRVana kits (Ambion Inc., Austin, TX, USA) and the TaqMan microRNA Reverse Transcription kit (Applied Biosystems, Foster City, CA, USA) were used to measure miRNAs as per the manufacturer's instructions. Relative quantification of the gene expression was performed using the $2^{-\Delta \Delta C t}$ method as previously described (25). U6 (miRNA) or GAPDH (mRNA) was validated as the internal control.

Western blot analysis. Cell lysates from different experiments were prepared using RIPA buffer, and the protein concentration was measured using the Bradford assay. Protein $(20 \mu \mathrm{g})$ was separated by $12 \%$ SDS-PAGE and transferred to nitrocellulose membranes (Amersham, Little Chalfont, UK). The membrane was then blocked with $2 \%$ non-fat dry milk at room temperature and incubated in primary antibodies at $4^{\circ} \mathrm{C}$ overnight. Anti-phospho-JAK2 (D4A8) antibody, anti-total JAK2 (D2E12) antibody, anti-phospho-STAT3 (D3A7) and anti-total STAT3 (D3Z2G) at a 1:1,000 diltuion were purchased from Cell Signaling Technology (Danvers, MA, USA). Anti-cleaved caspase-3 antibody at a 1:500 dilution, anti-survivin antibody at a 1:600 dilution, anti-XIAP antibodies at a 1:800 dilution and anti-GAPDH antibody at a 1:1,000 dilution were purchased from Santa Cruz Biotechnology, Inc. (Santa Cruz, CA, USA). The membrane was subsequently incubated in perox- 
idase-conjugated secondary antibody (Boster Corporation, Wuhan, Hubei, China) at a 1:1,000 dilution for $1 \mathrm{~h}$. The protein bands were visualized using an enhanced chemiluminescence detection system (Amersham).

Cell viability assay. Cell growth and viability were analyzed by an MTT assay according to standard protocols. Briefly, the cells were seeded in 96-well plates and miR-216a or anti-miR-216a mimic at a final concentration of $20 \mathrm{nmol} / \mathrm{l}$ was added and incubated for 24, 48, 72 and $96 \mathrm{~h}$. The previously applied medium was discarded, and fresh medium containing MTT $[5 \mathrm{mg} / \mathrm{ml}$ diluted in phosphate-buffered saline (PBS)] was added and incubated for $4 \mathrm{~h}$. Dimethyl sulfoxide was used to dissolve the formazan for $15 \mathrm{~min}$. Absorbance at $490 \mathrm{~nm}$ was measured using an ELISA reader (BioTek, Winooski, VT, USA).

Apoptosis assay. Cell apoptosis was detected using Annexin V/PI apoptosis kit (Invitrogen) according to the manufacturer's instructions. Briefly, the cells were harvested, washed with ice-cold PBS, and resuspended in binding buffer. Subsequently, the cells were incubated with $10 \mu \mathrm{l}$ of Annexin V stock solution at $4^{\circ} \mathrm{C}$ for $30 \mathrm{~min}$. PI $(5 \mu \mathrm{l})$ was then added and incubated for $5 \mathrm{~min}$. The cells were analyzed by a FACS analyzer (BD Biosciences, San Jose, CA, USA).

Xenograft tumor growth. The PANC-1 pancreatic cancer cell line was transfected with lentiviral vectors containing miR216a or anti-miR-216a (GenePharma). The transfected cells $\left(1 \times 10^{7}\right)$ were suspended in PBS and injected subcutaneously into the left groin. The length and width of the tumor were measured every 5 days, and the volume was calculated as: length $\mathrm{x}$ width $2 \times \pi / 6$. At 40 days after cell inoculation, the mice were anesthetized by subcutaneous injection of sodium pentobarbital $(40 \mathrm{mg} / \mathrm{kg})$. The tumor weight was assessed, and the total RNA and protein were extracted for subsequent analysis.

Statistical analysis. Data were presented as the means \pm standard deviation (SD). Comparisons between two groups were determined by the Student's t-test, and among groups using one-way ANOVA followed by Bonferroni post-hoc test. $\mathrm{P}<0.05$ was considered to indicate a statistically significant result. The data were analyzed using SPSS version 11.5 (SPSS Inc., Chicago, IL, USA).

\section{Results}

$J A K 2$ is a target gene of $m i R-216 a$. Previous studies demonstrated that miR-216a was significantly downregulated in pancreatic cancer (15-17). However, the function of miR-216 in pancreatic cancer remains to be determined. To investigate the biological role of miR-216a in pancreatic cancer, we used bioinformatic algorithms to screen the putative target. JAK2, which is involved in many cancer types, including pancreatic cancer, was found to be a target gene of miR-216a (Fig. 1A). To verify whether miR-216a regulated JAK2 expression by directly interacting with JAK2 mRNA, we cloned the 3'-UTR of human JKA2 downstream of the luciferase reporter gene in pGL3 plasmid. HEK293 cells were co-transfected with pGL3-
JAK2-3'-UTR and miR216a mimics. The results showed that miR216a mimics significantly $(\mathrm{p}<0.01)$ decreased the luciferase activity compared with the control, whereas miR216a mimics had no effect on the pGL3-mut-JAK2 3'-UTR transfected cells (Fig. 1B). The results suggested that miR-216a directly bound to 3'-UTR of JAK2.

miR-216a downregulates JAK2 expression in pancreatic cancer cells. To verify the interaction of miR-216a with JAK2, pancreatic cancer cells were transfected with miR-216a mimics or anti-miR-216a mimics and the expression of JAK2 was analyzed. The results showed that miR-216a significantly downregulated JAK2 mRNA levels, whereas anti-miR-216a markedly upregulated JAK2 mRNA levels in PANC-1 and Capan-2 cells (Fig. 2A). These results were confirmed by the western blot analysis, which revealed that phosphorylated JAK2 and total JAK2 protein levels were decreased in miR-216a-transfected pancreatic cancer cells. By contrast, anti-miR-216a increased phosphorylated JAK2 and total JAK2 protein levels in anti-miR-216a-transfected PANC-1 (Fig. 2B) and Capan-2 cells (Fig. 2C). The phosphorylation of STAT3, the downstream gene of JAK2, was also inhibited by miR-216a or increased by anti-miR-216a transfection, whereas the total levels of STAT3 were unaffected (Fig. 2B and C). These data indicated that miR-216a directly targeted JAK2 in pancreatic cancer cells.

miR-216a suppresses cell growth and viability in pancreatic cancer cells. Since JAK2 is directly targeted by miR-216a, we investigated next whether miR-216a is able to disturb the cell growth and viability of pancreatic cancer cells. Compared with the scramble miRNA-transfected group, miR-216a mimics significantly inhibited the cell growth and viability of PANC1 cells, whereas anti-miR-216a markedly enhanced cell growth and viability (Fig. 3A). The same effect was observed in Capan-2 cells (Fig. 3B).

miR-216a facilitates cell apoptosis of pancreatic cancer cells. To determine whether miR-216a was involved in the progression of pancreatic cancer, we assessed its effect on cell apoptosis. Fig. 4A shows that miR-216a significantly increased apoptosis of PANC-1 cells, whereas anti-216a inhibited cell apoptosis, compared with the control group (Fig. 4A). To verify the inhibitory effect of miR-216a on cell apoptosis, we measured the expression alterations of survivin and XIAP, the downstream gene of JAK2/STAT3 that inhibited cell apoptosis. The western blot analysis demonstrated that miR-216a transfection downregulated the protein expression levels of survivin and XIAP, which were enhanced by anti-miR-216a transfection. The pro-apoptotic protein cleaved caspase-3 was also increased by miR-216a transfection and decreased by anti-miR-216a transfection (Fig. 4B).

miR-216a represses xenograft tumor growth in vivo. To gain insight into the effect of miR-216a on pancreatic cancer, we inoculated LV-miR-216a-infected PANC-1 cells into nude mice and measured the effect of miR-216a expression on xenograft tumor growth. We initially detected the expression of miR-216a in xenograft tumor tissues. The results showed that LV-miR216a-infected cancer cells exhibited an increase of miR-216a 

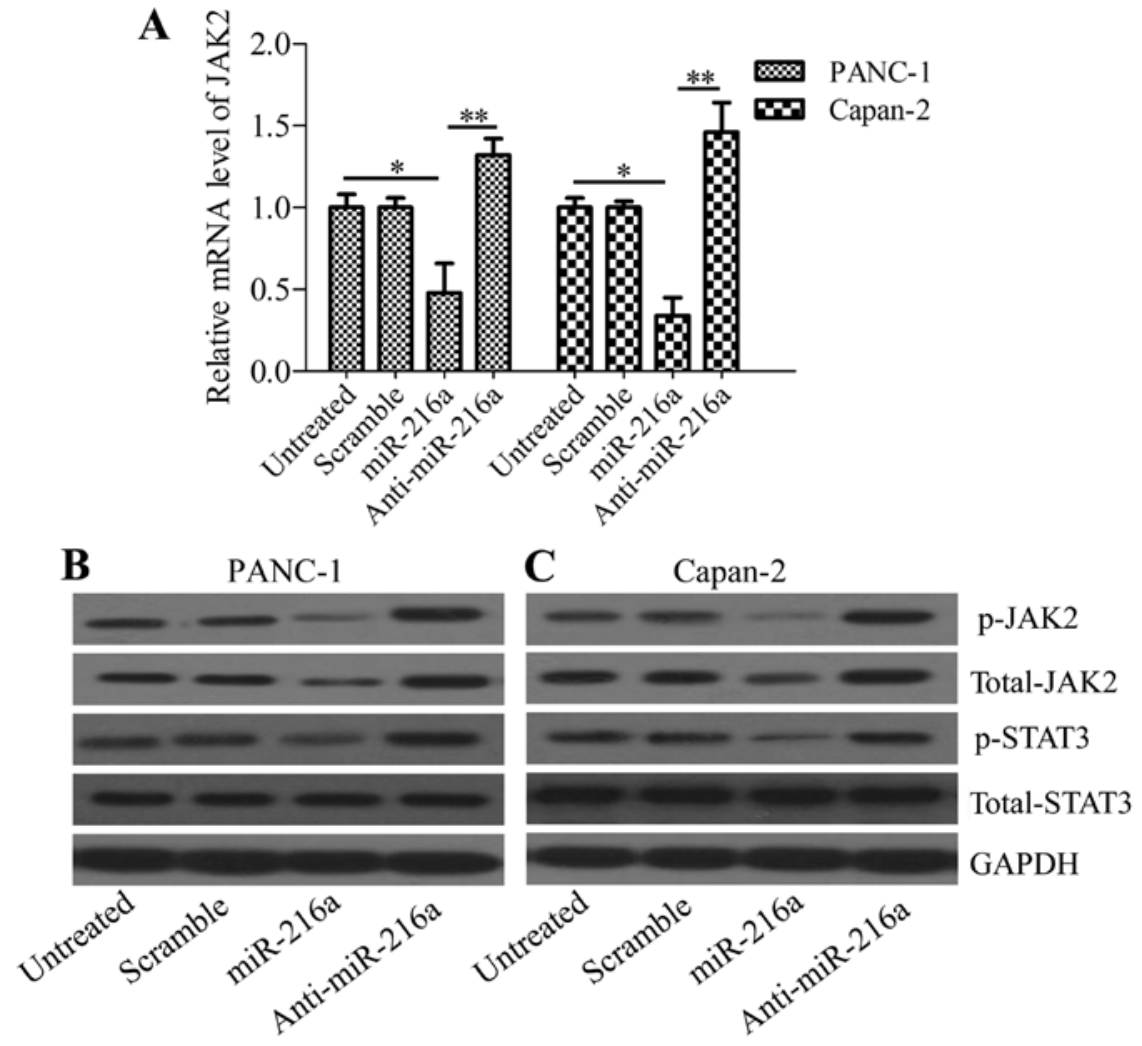

Figure 2. Effect of miR-216a on JAK2 expression in pancreatic cancer cells. (A) RT-qPCR was performed to detect the mRNA levels of JAK2 in pancreatic cancer cells. PANC-1 and Capan-2 cells were transfected with miR-216a or anti-216a mimics at a final concentration of $20 \mathrm{nmol} / 1 \mathrm{and}$ incubated for $48 \mathrm{~h}$. Untreated cells or cells transfected with scramble miRNA served as the control $\left({ }^{* *} \mathrm{p}<0.01\right.$ and $\left.{ }^{*} \mathrm{p}<0.05\right)$. Western blot analysis was performed to detect protein expression levels with indicated antibodies in miR-216a or anti-216a mimics transfected with (B) PANC-1 or (C) Capan-2 cells. JAK2, Janus kinase 2.
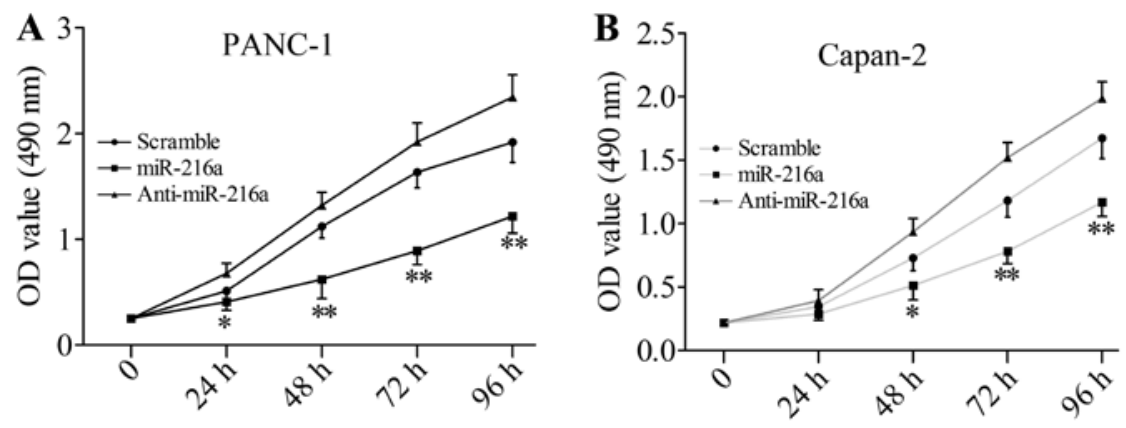

Figure 3. Effect of miR-216a on the cell growth and viability of pancreatic cancer cells. MTT assay was performed to determine the effect of miR-216a on the cell growth and viability of (A) PANC-1 and (B) Capan-2 cells. Pancreatic cancer cells were transfected with scramble miRNA, miRNA-216a mimics or anti-miRNA-216a mimics for $24,48,72$ and $96 \mathrm{~h}$. Data are presented as mean \pm SD for three independent experiments $\left({ }^{* *} \mathrm{p}<0.01\right.$ and $\left.{ }^{*} \mathrm{p}<0.05\right)$.
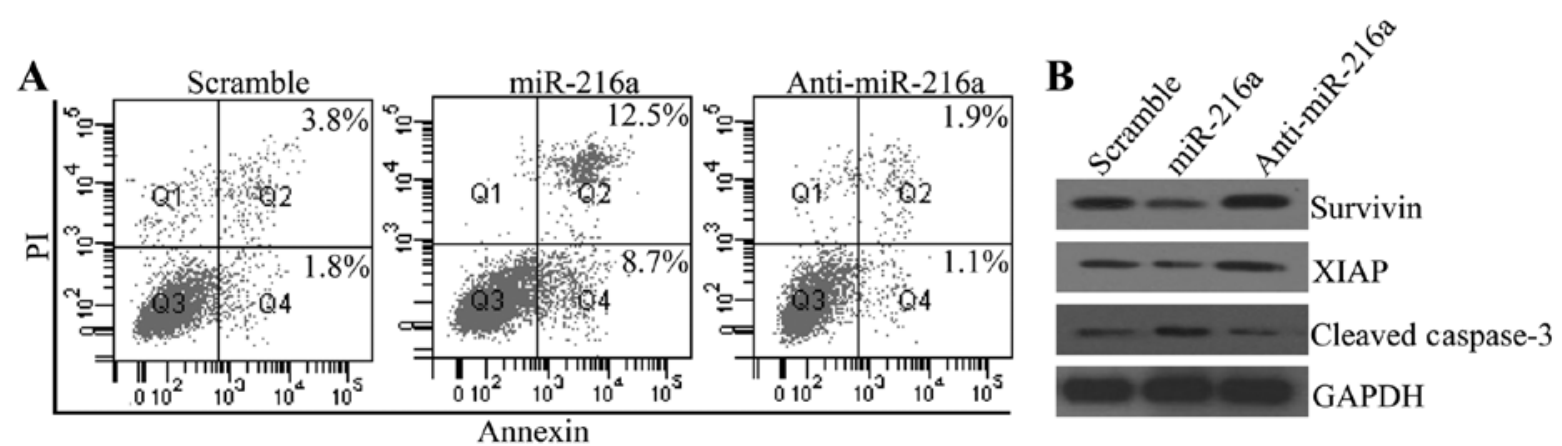

Figure 4. Effect of miR-216a on cell apoptosis. (A) Apoptotic cells were detected by Annexin V/PI assay. PANC-1 cells were transfected with miR-216a or anti-miR-216a mimics for $48 \mathrm{~h}$. (B) Western blot analysis was used to determine the apoptotic-associated protein expression levels with indicated antibodies. 

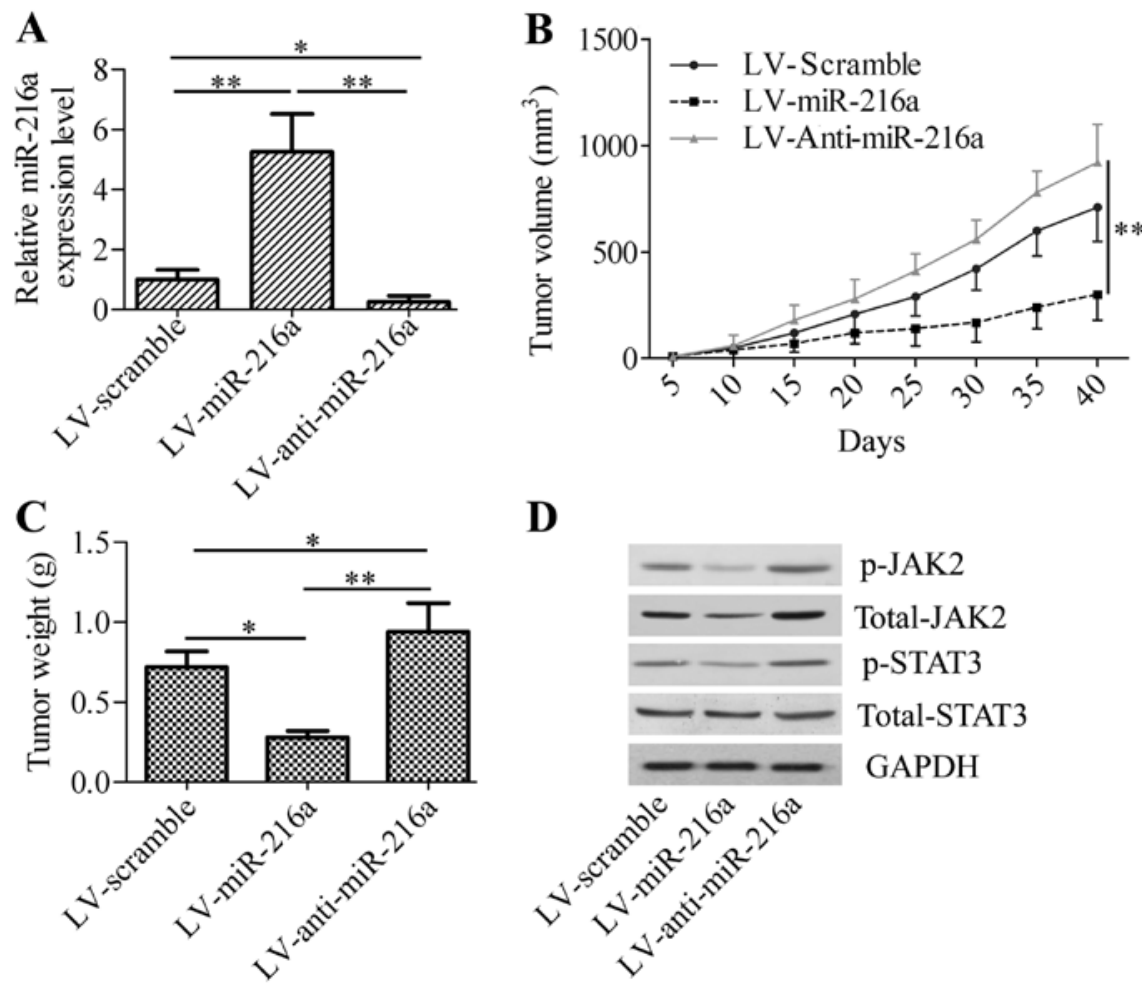

Figure 5. Effect of miR-216a on pancreatic cancer in vivo. (A) RT-qPCR was performed to determine the expression levels of miR-216a in xenograft tumor tissues. (B) Tumor volumes of each group were measured every five days during the tumor growth process. (C) Tumor weight was measured from the xenograft model. Cells were infected with LV-scramble, LV-miR-216a or LV-anti-miR-216a and inoculated into nude mice. At 40 days after cell inoculation, xenograft tumor was isolated. The weight was measured and the protein and total RNA were extracted for (D) the western blot analysis. * $\mathrm{P}<0.05$ and ${ }^{* *} \mathrm{p}<0.01$.

levels in xenograft tumor tissues, whereas a significant decrease of miR-216a levels was observed in the LV-anti-miR-216atreated group (Fig. 5A). We determined whether miR-216a overexpression or silencing affects xenograft tumor growth. LV-miR-216a markedly decreased xenograft tumor growth, including tumor volume and weight (Fig. 5B and C), whereas LV-anti-miR-216a significantly increased xenograft tumor growth compared with the control group. Western blot analysis revealed that the phosphorylated JAK2 and total JAK2 protein levels were decreased in the LV-miR-216a xenograft tumor, whereas phosphorylated JAK2 and total JAK2 protein levels were increased in LV-anti-miR-216a xenograft tumor. The phosphorylation of STAT3, the downstream gene of JAK2, was also significantly downregulated in the LV-miR-216a xenograft tumor and upregulated in the LV-anti-miR-216a xenograft tumor. However, LV-miR-216a or LV-anti-miR-216a exhibited no effect of total STAT3 (Fig. 5D). These results confirmed the tumor-suppressor activity of miR-216a on pancreatic cancer cell growth by targeting and regulating JAK2.

\section{Discussion}

Previous studies have demonstrated that the expression of miR-216a is significantly decreased in pancreatic cancer patients (15) or a murine model of pancreatic cancer (26). Link et al suggested that miR-216a downregulation in feces may be used as fecal miRNA biomarkers for pancreatic cancer (16). Decreased miR-216a expression is also verified in pancreatic intraepithelial neoplasms (17). The abovementioned studies suggest that miR-216a may be used as a diagnostic marker and as a target for pancreatic cancer. However, the potential precise mechanism of miR-216a in regulating pancreatic cancer remains to be determined. To the best of our knowledge, the present study is the first to provide evidence that miR-216a regulates pancreatic cancer by directly targeting 3'-UTR of JAK2.

miR-216a is a member of the miR-216 family, which is widely conserved in many species (27). The function of miR-216a is not well delineated. miR-216a together with miR-217 is induced by transforming growth factor- $\beta$ target phosphatase and tensin homologue, leading to Akt activation in glomerular mesangial cells in kidney disorders (28). miR-216a targets YB-1, which mediates Tsc-22 post-transcriptional regulation, having an important function in the pathogenesis of diabetic nephropathy (29). Jeyapalan et al found that miR-216 suppresses tumorigenesis and angiogenesis by targeting 3'-UTR of CD44 (27). In early hepatocarcinogenesis, miR216a, elevated by the androgen pathway, inhibits the tumor suppressor in lung cancer-1 (30). Increased miR-216a level is considered a biomarker of pancreatitis $(31,32)$. Thus, miR-216a apparently has a wide biological effect on different conditions.

In the present study, we identified that miR-216a interacted with JAK2 to negatively regulate pancreatic cancer. Forced expression of miR-216a significantly inhibited cell growth and promoted the cell apoptosis of pancreatic cancer cells. The JAK2/STAT3 signaling pathway was markedly disturbed by miR-216a. The aberrant activation of the JAK2/STAT3 signaling pathway is considered to be extensively involved in tumorigenesis (33). In recent years, the JAK2V617F mutation has been found to be involved in myeloproliferative 
disorders, in which the 617 th amino acid mutation leads to the sustained activation of JAK2 and STAT3 (34). Disruption of JAK2/STAT3 leads to the inhibition of gastric carcinogenesis (35). The JAK2/STAT3 signaling pathway is also involved in pancreatic cancer. STAT3 is a critical factor in Kras-induced pancreatic cancers (36). Disruption of STAT3 in the transgenic pancreas profoundly suppresses the pancreatic metaplasia (37). NADPH oxidase promotes pancreatic cancer by maintaining the sustained phosphorylation of JAK2 by tyrosine phosphatases (38). Inhibition of JAK2 and Notch together has a better effect than single inhibitions on pancreatic cell progression (39). Activated STAT3 induces the elevation of anti-apoptotic genes $(40,41)$. In the present study, we found that miR-216a inhibited the phosphorylation of STAT3, and the downstream anti-apoptotic genes survivin and XIAP were downregulated, leading to the apoptosis of pancreatic cancer cells. Thus, targeting JAK2/STAT3 has potential for the treatment of pancreatic cancer.

Application of miRNAs in the treatment of cancer has achieved convincing antitumor effects in animal models (42). An increasing number of studies have been performed to develop potential miRNAs in the treatment of pancreatic cancer. miR-219-1-3p is a negative regulator of the mucin MUC4 expression and inhibits the progression of pancreatic cancer (43). miRNA-141 exerts an inhibitory effect on cell proliferation and invasion by directly targeting MAP4K4 (44). Miao et al revealed that miR-203 suppresses cancer cell migration and invasion by targeting caveolin-1 in pancreatic cancer (45). More recently, miR-196a has been found to bind nuclear factor $\kappa$-B-inhibitor $\alpha$ to facilitate pancreatic cancer progression (46). miR let-7 blocks STAT3 activation by JAK2 by increasing SOCS3 expression in pancreatic cancer cells (47). miR-375 significantly downregulates in pancreatic cancer (48) targeting JAK2/STAT3 to suppress gastric carcinogenesis (35), suggesting an inhibitory role of miR-375 on JAK2 in pancreatic cancer cells. In the present study, we provided evidence that miR-216a, which is frequently downregulated in pancreatic cancer, directly targeted JAK2 to inhibit pancreatic cancer growth in vitro and in vivo. A recent study has demonstrated that miR-216a targeting beclin 1, an autophagy-related gene, inhibits cell autophagy in endothelial cells, suggesting a potential function in the pathogenesis in cardiovascular disorders (49). More investigations are required to determine whether miR-216a is associated with autophagy in pancreatic cancer cells due to the important function of autophagy in pancreatic cancer development (50).

In summary, to the best of our knowledge, this is the first study to demonstrate the direct interaction between miR-216a and JAK2, and the regulation effect of miR-216a on JAK2 in pancreatic cancer cells. Considering the tumorigenic function of JAK2 in pancreatic cancer, targeting JAK2/STAT3 signaling pathway by miR-126a provides novel insight for the development of useful therapeutic strategy against pancreatic cancer.

\section{References}

1. Vincent A, Herman J, Schulick R, Hruban RH and Goggins M: Pancreatic cancer. Lancet 378: 607-620, 2011.

2. Kleeff J, Michalski CW, Friess H and Büchler MW: Surgical treatment of pancreatic cancer: the role of adjuvant and multimodal therapies. Eur J Surg Oncol 33: 817-823, 2007.
3. Burris HA III, Moore MJ, Andersen J, et al: Improvements in survival and clinical benefit with gemcitabine as first-line therapy for patients with advanced pancreas cancer: a randomized trial. J Clin Oncol 15: 2403-2413, 1997.

4. Poplin E, Feng Y, Berlin J, et al: Phase III, randomized study of gemcitabine and oxaliplatin versus gemcitabine (fixed-dose rate infusion) compared with gemcitabine (30-minute infusion) in patients with pancreatic carcinoma E6201: a trial of the Eastern Cooperative Oncology Group. J Clin Oncol 27: 3778-3785, 2009.

5. Heinemann V, Quietzsch D, Gieseler F, et al: Randomized phase III trial of gemcitabine plus cisplatin compared with gemcitabine alone in advanced pancreatic cancer. J Clin Oncol 24: 3946-3952, 2006.

6. Conroy T, Desseigne F, Ychou M, et al: FOLFIRINOX versus gemcitabine for metastatic pancreatic cancer. N Engl J Med 364: 1817-1825, 2011.

7. Schneider G and Schmid RM: Genetic alterations in pancreatic carcinoma. Mol Cancer 2: 15, 2003.

8. Hezel AF, Kimmelman AC, Stanger BZ, Bardeesy N and Depinho RA: Genetics and biology of pancreatic ductal adenocarcinoma. Genes Dev 20: 1218-1249, 2006.

9. Chiu J and Yau T: Metastatic pancreatic cancer: are we making progress in treatment? Gastroenterol Res Pract 2012: 898931, 2012.

10. Mendell JT and Olson EN: MicroRNAs in stress signaling and human disease. Cell 148: 1172-1187, 2012.

11. Bartel DP: MicroRNAs: genomics, biogenesis, mechanism, and function. Cell 116: 281-297, 2004.

12. Winter J, Jung S, Keller S, Gregory RI and Diederichs S: Many roads to maturity: microRNA biogenesis pathways and their regulation. Nat Cell Biol 11: 228-234, 2009.

13. Lujambio A and Lowe SW: The microcosmos of cancer. Nature 482: 347-355, 2012.

14. Drakaki A and Iliopoulos D: MicroRNA-gene signaling pathways in pancreatic cancer. Biomed J 36: 200-208, 2013.

15. Hou B, Jian Z, Chen S, et al: Expression of miR-216a in pancreatic cancer and its clinical significance. Nan Fang Yi Ke Da Xue Xue Bao 32: 1628-1631, 2012 (In Chinese).

16. Link A, Becker V, Goel A, Wex T and Malfertheiner P: Feasibility of fecal microRNAs as novel biomarkers for pancreatic cancer. PLoS One 7: e42933, 2012.

17. Yu J, Li A, Hong SM, Hruban RH and Goggins M: MicroRNA alterations of pancreatic intraepithelial neoplasias. Clin Cancer Res 18: 981-992, 2012.

18. Scholz A, Heinze S, Detjen KM, et al: Activated signal transducer and activator of transcription 3 (STAT3) supports the malignant phenotype of human pancreatic cancer. Gastroenterology 125: 891-905, 2003.

19. Zhong Z, Wen Z and Darnell JE Jr: Stat3: a STAT family member activated by tyrosine phosphorylation in response to epidermal growth factor and interleukin-6. Science 264: 95-98, 1994.

20. Shuai K, Horvath CM, Huang LH, et al: Interferon activation of the transcription factor Stat91 involves dimerization through SH2-phosphotyrosyl peptide interactions. Cell 76: 821-828, 1994.

21. Chan KS, Sano S, Kiguchi K, et al: Disruption of Stat3 reveals a critical role in both the initiation and the promotion stages of epithelial carcinogenesis. J Clin Invest 114: 720-728, 2004.

22. Wang T, Niu G, Kortylewski M, et al: Regulation of the innate and adaptive immune responses by Stat-3 signaling in tumor cells. Nat Med 10: 48-54, 2004.

23. Frank DA: STAT3 as a central mediator of neoplastic cellular transformation. Cancer Lett 251: 199-210, 2007.

24. Kumar C, Purandare AV, Lee FY and Lorenzi MV: Kinase drug discovery approaches in chronic myeloproliferative disorders. Oncogene 28: 2305-2313, 2009.

25. Livak KJ and Schmittgen TD: Analysis of relative gene expression data using real-time quantitative PCR and the $2^{-\triangle \Delta C \mathrm{~T}}$ method. Methods 25: 402-408, 2001.

26. Ali S, Banerjee S, Logna F, et al: Inactivation of Ink4a/Arf leads to deregulated expression of miRNAs in K-Ras transgenic mouse model of pancreatic cancer. J Cell Physiol 227: 3373-3380, 2012.

27. Jeyapalan Z, Deng Z, Shatseva T, et al: Expression of CD44 3'-untranslated region regulates endogenous microRNA functions in tumorigenesis and angiogenesis. Nucleic Acids Res 39: 3026-3041, 2011.

28. Kato M, Putta S, Wang M, et al: TGF- $\beta$ activates Akt kinase through a microRNA-dependent amplifying circuit targeting PTEN. Nat Cell Biol 11: 881-889, 2009. 
29. Kato M, Wang L, Putta S, et al: Post-transcriptional up-regulation of Tsc- 22 by Ybx1, a target of miR-216a, mediates TGF- $\beta$ induced collagen expression in kidney cells. J Biol Chem 285 34004-34015, 2010.

30. Chen PJ, Yeh SH, Liu WH, et al: Androgen pathway stimulates microRNA-216a transcription to suppress the tumor suppressor in lung cancer-1 gene in early hepatocarcinogenesis. Hepatology 56 632-643, 2012.

31. Usborne AL, Smith AT, Engle SK, et al: Biomarkers of exocrine pancreatic injury in 2 rat acute pancreatitis models. Toxicol Pathol 42: 195-203, 2014

32. Blenkiron C, Askelund KJ, Shanbhag ST, et al: MicroRNAs in mesenteric lymph and plasma during acute pancreatitis. Ann Surg: Feb 6, 2014 (Epub ahead of print).

33. Yu H, Pardoll D and Jove R: STATs in cancer inflammation and immunity: a leading role for STAT3. Nat Rev Cancer 9: 798-809, 2009.

34. Gabler K, Behrmann I and Haan C: JAK2 mutants (e.g. JAK2V617F) and their importance as drug targets in myeloproliferative neoplasms. JAKSTAT 2: e25025, 2013.

35. Miao L, Liu K, Xie M, Xing Y and Xi T: miR-375 inhibits Helicobacter pylori-induced gastric carcinogenesis by blocking JAK2-STAT3 signaling. Cancer Immunol Immunother 63 : 699-711, 2014

36. Corcoran RB, Contino G, Deshpande V, et al: STAT3 plays a critical role in $K R A S$-induced pancreatic tumorigenesis. Cancer Res 71: 5020-5029, 2011

37. Miyatsuka T, Kaneto H, Shiraiwa T, et al: Persistent expression of PDX-1 in the pancreas causes acinar-to-ductal metaplasia through Stat3 activation. Genes Dev 20: 1435-1440, 2006.

38. Lee JK,Edderkaoui M, Truong P, et al: NADPH oxidase promotes pancreatic cancer cell survival via inhibiting JAK2 dephosphorylation by tyrosine phosphatases. Gastroenterology 133 : $1637-1648,2007$

39. Palagani V, Bozko P, El Khatib M, et al: Combined inhibition of Notch and JAK/STAT is superior to monotherapies and impairs pancreatic cancer progression. Carcinogenesis 35: 859-866, 2014.
40. Butturini E, Carcereri de Prati A, Chiavegato G, et al: Mild oxidative stress induces S-glutathionylation of STAT3 and enhances chemosensitivity of tumoural cells to chemotherapeutic drugs. Free Radic Biol Med 65: 1322-1330, 2013.

41. Kim JK, Kim JY, Kim HJ, et al: Scoparone exerts anti-tumor activity against DU145 prostate cancer cells via inhibition of STAT3 activity. PLoS One 8: e80391, 2013.

42. Ørom UA, Kauppinen S and Lund AH: LNA-modified oligonucleotides mediate specific inhibition of microRNA function. Gene 372: 137-141, 2006.

43. Lahdaoui F, Delpu Y, Vincent A, et al: miR-219-1-3p is a negative regulator of the mucin MUC4 expression and is a tumor suppressor in pancreatic cancer. Oncogene 0: 1-9, 2014.

44. Zhao G, Wang B, Liu Y, et al: miRNA-141, downregulated in pancreatic cancer, inhibits cell proliferation and invasion by directly targeting MAP4K4. Mol Cancer Ther 12: 2569-2580, 2013.

45. Miao L, Xiong X, Lin Y, et al: miR-203 inhibits tumor cell migration and invasion via caveolin-1 in pancreatic cancer cells. Oncol Lett 7: 658-662, 2014.

46. Huang F, Tang J, Zhuang X, et al: MiR-196a promotes pancreatic cancer progression by targeting nuclear factor kappa-B-inhibitor alpha. PLoS One 9: e87897, 2014.

47. Patel K, Kollory A, Takashima A, et al: MicroRNA let-7 downregulates STAT3 phosphorylation in pancreatic cancer cells by increasing SOCS3 expression. Cancer Lett 347: 54-64, 2014.

48. Song S, Zhou J, He S, et al: Expression levels of microRNA-375 in pancreatic cancer. Biomed Rep 1: 393-398, 2013.

49. Menghini R, Casagrande V, Marino A, et al: MiR-216a: a link between endothelial dysfunction and autophagy. Cell Death Dis 5: e1029, 2014.

50. Yang S, Wang X, Contino G, et al: Pancreatic cancers require autophagy for tumor growth. Genes Dev 25: 717-729, 2011. 